\title{
Association of RASSF1A, DCR2, and CASP8 Methylation with Survival in Neuroblastoma: A Pooled Analysis Using Reconstructed Individual Patient Data
}

\author{
Waleed M. Hassan $\mathbb{D}^{1,2}$ Mohamed S. Bakry, ${ }^{2}$ Timo Siepmann $\mathbb{D}^{1,3}$ and Ben Illigens ${ }^{4}$ \\ ${ }^{1}$ Dresden International University, Division of Health Care Sciences, Center for Clinical Research and Management Education, \\ Dresden 01067, Germany \\ ${ }^{2}$ Research Department, Children's Cancer Hospital Egypt 57357, Cairo 11588, Egypt \\ ${ }^{3}$ Department of Neurology, University Hospital Carl Gustav Carus, Technische Universität Dresden, Dresden 01307, Germany \\ ${ }^{4}$ Department of Neurology, Beth Israel Deaconess Medical Center, Harvard Medical School, Boston, MA 02215, USA
}

Correspondence should be addressed to Timo Siepmann; timo.siepmann@ukdd.de

Received 22 August 2020; Revised 9 October 2020; Accepted 17 October 2020; Published 15 December 2020

Academic Editor: Somchai Chutipongtanate

Copyright (c) 2020 Waleed M. Hassan et al. This is an open access article distributed under the Creative Commons Attribution License, which permits unrestricted use, distribution, and reproduction in any medium, provided the original work is properly cited.

\begin{abstract}
Neuroblastoma (NB) is a heterogeneous tumor affecting children. It shows a wide spectrum of clinical outcomes; therefore, development of risk stratification is critical to provide optimum treatment. Since epigenetic alterations such as DNA methylation have emerged as an important feature of both development and progression in NB, in this study, we aimed to quantify the effect of methylation of three distinct genes (RASSF1A, DCR2, and CASP8) on overall survival in NB patients. We performed a systematic review using PubMed, Embase, and Cochrane libraries. Individual patient data was retrieved from extracted Kaplan-Meier curves. Data from studies was then merged, and analysis was done on the full data set. Seven studies met the inclusion criteria. Methylation of the three genes had worse overall survival than the unmethylated arms. Five-year survival for the methylated arm of RASSF1A, DCR2, and CASP8 was $63.19 \%$ (95\% CI 56.55-70.60), 57.78\% (95\% CI 47.63-70.08), and 56.39\% (95\% CI 49.53-64.19), respectively, while for the unmethylated arm, it was 93.10\% (95\% CI 87.40-99.1), 84.84\% (95\% CI 80.04-89.92), and 83.68\% (95\% CI 80.28-87.22), respectively. In conclusion, our results indicate that in NB patients, RASSF1A, DCR2, and CASP8 methylation is associated with poor prognosis. Large prospective studies will be necessary to confirm definitive correlation between methylation of these genes and survival taking into account all other known risk factors. (PROSPERO registration number CRD42017082264).
\end{abstract}

\section{Introduction}

Neuroblastoma (NB) is a heterogeneous tumor affecting the normal development of the adrenal medulla and paravertebral sympathetic ganglia in early childhood $[1,2]$. The age-standardized rate of NB is 10.4 per million personyears in children aged $0-14$ based on data from the largest cancer registry study including 153 registries from 62 countries [3]. The peak incidence age of NB is in the first year, with very few cases reported after the age of 10 years [4-6]. $\mathrm{NB}$ is considered a major cause of pediatric cancer mortality accounting for around $15 \%$ of all deaths [7].
The overall impact of known environmental exposures on the etiology of NB is very low, and the consistent incidence rates of neuroblastoma in children across different regions in the world support the hypothesis of a major role of genetic factors $[3,6]$. NB shows a wide spectrum of clinical outcomes, since most of the tumors can regress spontaneously while some may be aggressive $[8,9]$. This is mainly caused by the difference in biological and molecular features. Hence, NB risk stratification to predict clinical outcomes is continuously evolving [10]. Many earlier studies were aimed at assessing the prognostic power of different genetic mutations in NB [2]. 
The most common factors associated with the outcome and currently involved in determining the risk of patients are age at diagnosis, stage at diagnosis, MYCN amplification, histopathological classification, and DNA ploidy status [1014]. The use of these variables in risk stratification has led to defining two subgroups. The first is the low-risk group in which most of the tumors regress spontaneously or with minimal treatment. The second is the high-risk group in which most of the tumors have unfavorable outcomes and will require intensive therapy. Not all of the patients in each group show the same pattern of the disease. Moreover, there are some less well-defined groups between both groups. This may cause some of the patients to be overtreated or undertreated [15-18]. Therefore, additional prognostic factors may be needed in order to refine the risk stratification of NB $[15,19]$.

Epigenetic alterations such as DNA methylation have emerged as an important feature of both development and progression in many cancers. Methylation of promoter $\mathrm{CpG}$ islands is known to inhibit transcriptional initiation and cause permanent silencing of downstream genes [20]. The most common known epigenetic alterations associated with the outcome in NB are methylation of RASSF1A, DCR2, and CASP8 genes [21-24].

In this study, we quantitatively reviewed the effect of DNA methylation of three distinct genes (RASSF1A, DCR2, and CASP8) and their relation with the outcome using individual reconstructed patient data.

\section{Methods}

2.1. Search Criteria. We searched available studies in PubMed, Embase, and Cochrane libraries through 1 October 2020 using the following search statement: (neuroblastoma OR nb OR nbl) AND (RASSF1A OR DCR2 OR CASP8 OR "decoy receptor 2 " OR "caspase 8 "). Studies were included if they met the following inclusion criteria: (1) studies were conducted on neuroblastoma patients, (2) overall survival curves were reported for at least one of the studied genes according to the methylation status, (3) results for each gene were reported separately, and (4) methylation status was measured from tumor samples. Two independent reviewers conducted the search. Discrepancies were resolved based on discussion.

2.2. Data Extraction. For each included paper, Kaplan-Meier (KM) curves for the included genes were extracted. The curves were digitized using the software developed by AmsterCHEM ScanIt [25]. ScanIt is a free digitizer program that is used to extract data from graphs by automatic tracing of lines and converting them to numerical coordinates. Individual patient data (IPD) was then reconstructed using an iterative algorithm developed by Guyot et al. [26]. The algorithm utilizes the coordinates extracted by the digitizer software and the total number at risk in each arm to solve the KM equations originally used to develop the graph. The algorithm assumes constant censoring (noninformative). Numbers at risk at different points will be used when reported to increase the accuracy of the algorithm.
2.3. Statistical Analysis. The code developed by Guyot et al. was used to retrieve IPD of each KM. Data retrieved from each KM was redrawn and checked visually if the original and the redrawn KM are similar. All the retrieved IPD data were then merged in one file. The aggregated data was then used to construct a KM for each gene. A chi-square logrank test was then performed to test the statistical significance of the survival difference between both arms. 5-year survival probability was then reported. A multivariable Cox proportional hazard model was used to determine the significance of methylation status adjusting to the paper from which the data has been extracted. The adjusted hazard ratio (HR) was reported with associated 95\% confidence intervals (CI). All the statistical analyses were done using $\mathrm{R}$ (version 3.4.3) [27].

This review protocol was registered in the Prospero database (CRD42017082264).

\section{Results}

We identified 750 potential papers from our search. The PRISMA (Preferred Reporting Items for Systematic Reviews and Meta-Analyses) statement was followed (Figure 1) There were seven included articles for which IPD for each gene was extracted. A summary of the IPD retrieved is displayed in Table 1. IPD is available in Supplementary Table 1.

3.1. RASSF1A. Data on RASSF1A for 254 patients from three different studies were included. Out of which, 182 (71.65\%) were methylated. The methylated arm had statistically significant worse OS than the unmethylated arm (log-rank test $p$ value $<0.0001)$. 5-year survival probability for the methylated arm was 63.19\% (95\% CI 56.55-70.60), while for the unmethylated arm, it was $93.10 \%$ (95\% CI 87.40-99.1). Figure 2 displays the KM of the aggregated data for the RASSF1A gene. Adjusted HR for the methylated arm was 4.36 (95\% CI 2.16-8.81).

3.2. DCR2. Data on DCR2 for 292 patients from three different studies were included. Out of which, $84(28.77 \%)$ were methylated. The methylated arm had statistically significant worse OS than the unmethylated arm (log-rank test $p$ value $<0.0001)$. 5-year survival probability for the methylated arm was 57.78\% (95\% CI 47.63-70.08), while for the unmethylated arm, it was $84.84 \%$ (95\% CI 80.04 89.92). Figure 3 displays the KM of the aggregated data for the DCR2 gene. Adjusted HR for the methylated arm was 3.53 (95\% CI 2.20-5.65).

3.3. CASP8. Data on CASP8 for 620 patients from five different studies were included. Out of which, 185 (29.84\%) were methylated. The methylated arm had statistically significant worse OS than the unmethylated arm (log-rank test $p$ value $<0.0001)$. 5-year survival probability for the methylated arm was $56.39 \%$ (95\% CI 49.53-64.19), while for the unmethylated arm, it was $83.68 \%$ (95\% CI 80.28-87.22). Figure 4 displays the KM of the aggregated data for the CASP8 gene. Adjusted HR for the methylated arm was 4.66 (95\% CI 3.42-6.35). 

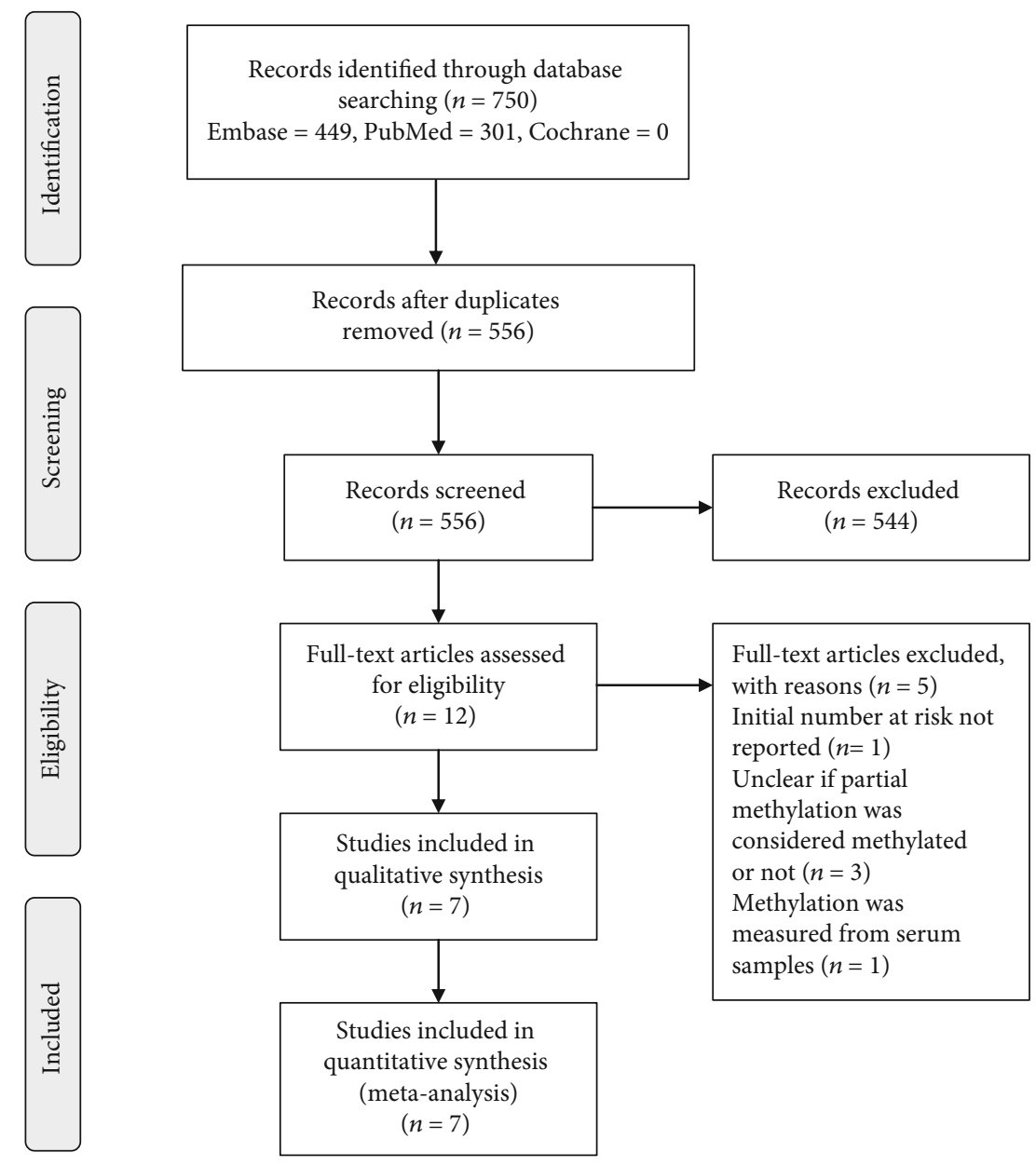

FiguRE 1: Summary of the search strategy performed to identify relevant studies to be included in the analysis.

\section{Discussion}

Carcinogenesis is thought to be a result of interaction of genetic and epigenetic alterations [28, 29]. Epigenetic alterations, by definition, comprise mitotically and meiotically heritable changes in gene expression that are not caused by changes in the primary DNA sequence [30]. Perhaps, DNA methylation is the most extensively studied epigenetic alteration $[28,31]$. DNA methylation is the addition of a methyl group 5 of the cytosine within the dinucleotide CpG. Hypermethylation of $\mathrm{CpG}$ islands, which are often present in gene promoters, leads to gene silencing. This has emerged as an important feature of both development and progression in many cancers $[20,22,32]$.

Current risk classification of NB patients is based mainly on age of onset, disease stage at diagnosis, and MYCN amplification. Although this risk classification has been significantly improved in recent years, undertreatment or overtreatment will still occur for certain children. This results in suboptimal survival rates for some patients in the low-risk group and exposes some patients in the high-risk group to unnecessarily risk for potential longterm side effects of the toxic therapy. Therefore, it is important to have additional biomarkers included in the current risk classification. Only then, patients will receive the most appropriate therapy, without putting them at risk of under- or overtreatment $[16,24]$.

The neuroblastoma genome displays distinct patterns of DNA methylation which may have a clinicopathologic value and hence can be associated with different risk groups [33, 34]. RASSF1A, DCR2, and CASP8 are the most studied genes for correlation between their methylation and survival outcome. Most of these previous studies included few numbers of patients. In this study, we quantitatively reviewed the effect of DNA methylation of these three genes (RASSF1A, DCR2, and CASP8) on survival in NB patients. This is the first metaanalysis to combine the results of these studies aimed at showing their potential as a prognostic marker in NB patients. Reconstructed IPD was used in this study.

4.1. RASSF1A. RASSF1A (Ras association domain family protein 1 isoform A) was described by Dammann et al. as a Ras effector located at 3p21.3 $[35,36]$. The RASSF1A gene encodes a protein like that of Ras effectors which exerts its function through a Ras signal transduction pathway. The RASSF1A induces growth arrest by inhibiting the accumulation of native cyclin D1 and preventing cells from passing through the retinoblastoma family cell cycle restriction point and entering the S phase $[21,24,36,37]$. Therefore, loss of expression or altered expression through methylation of this 


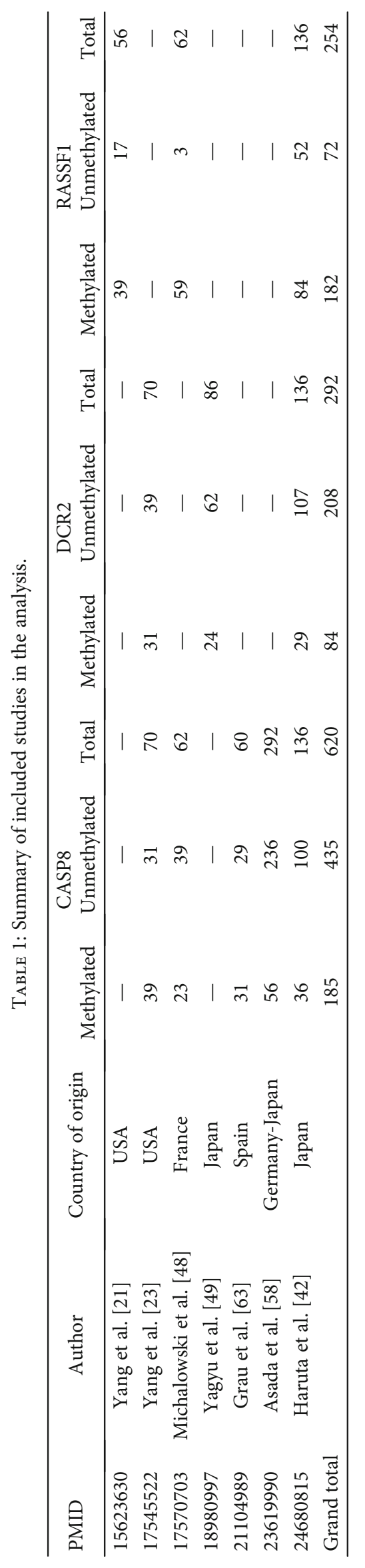




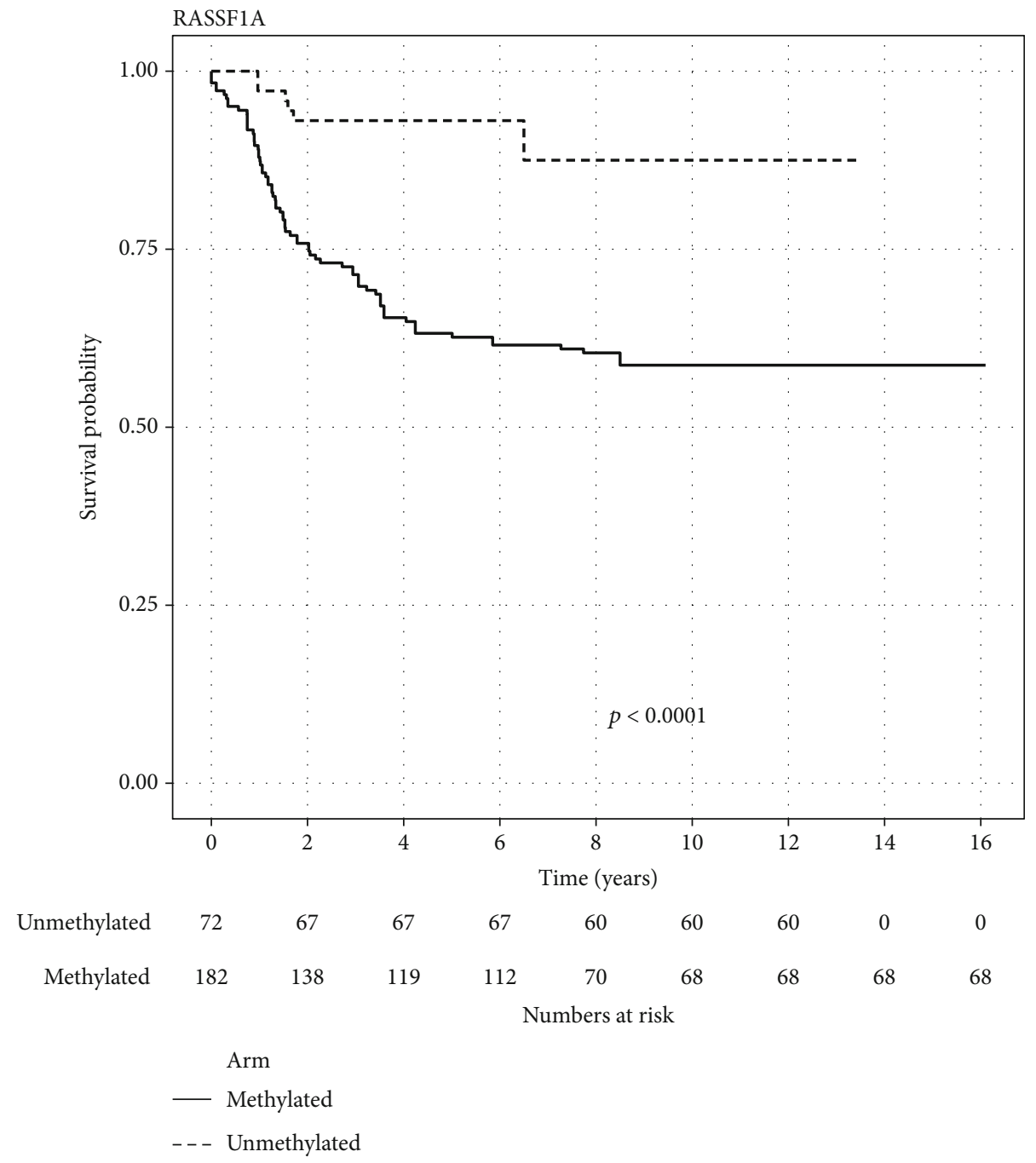

Figure 2: Reconstructed Kaplan-Meier graph of overall survival according to RASSF1A methylation using aggregated data.

gene has been associated with the pathogenesis of variety of cancers such as lung, breast, ovarian, and kidney cancers and pediatric tumors [36, 38-40].

In our study, RASSF1A was found methylated in $72 \%$ of NB patients; this was derived from the three studies included where methylation of RASSF1A ranged from $62 \%$ to $95 \%$ $[21,41,42]$. Our result is similar to previous studies not included in this analysis, which have found RASSF1A to be methylated in $50-100 \%$ of NB tumor specimens $[24,36,43$, 44]. Methylation of RASSF1A is less prevalent in serum samples compared to tumor samples. This was observed in one study comparing the RASSF1A methylation in serum with tumor samples revealing that RASSF1A was methylated in $17 / 68(25 \%)$ in serum samples versus 64/68 (94\%) in tumor samples of neuroblastoma patients [43]. Association between RASSF1A methylation and other known prognostic factors has been tested in many previous studies. One study reported that RASSF1A methylation was statistically significantly more prevalent in MYCN amplified tumors than in MYCN nonamplified tumors [44]. On the other hand, two studies (one of them is included in our meta-analysis) reported that there was no statistically significant difference in RASSF1A methylation prevalence according to MYCN amplification $[21,45]$. Two studies (one of them is included in our metaanalysis) concluded that RASSF1A methylation in NB patients was statistically significantly more prevalent in the age group $>1$ year, compared to the age group $<1$ year [21, 38]. The study included in our analysis also concluded that RASSF1A methylation was statistically significantly correlated with the risk group, as RASSF1A methylation was more prevalent in the high-risk group [21]. Another study stratified neuroblastoma patients according to whether the tumor sample was from a primary or a relapsed tumor. This study found that RASSF1A was methylated in all relapsed tumors $17 / 17(100 \%)$ and in $42 / 45$ (93\%) of primary tumors [44]. Further studies with a larger number of patients may be needed to confirm these associations between RASSF1A methylation and known risk factors.

In our study, 5-year survival probability for the RASSF1A methylated arm was 63\% (95\% CI 56.55-70.60), while for the unmethylated arm, it was $93.10 \%$ (95\% CI 87.40-99.1) (Figure 2) (log-rank $p$ value $<0.0001)$. Adjusted HR for the methylated arm was 4.36 (95\% CI 2.16-8.81). These results are consistent with the results in some of the included studies 


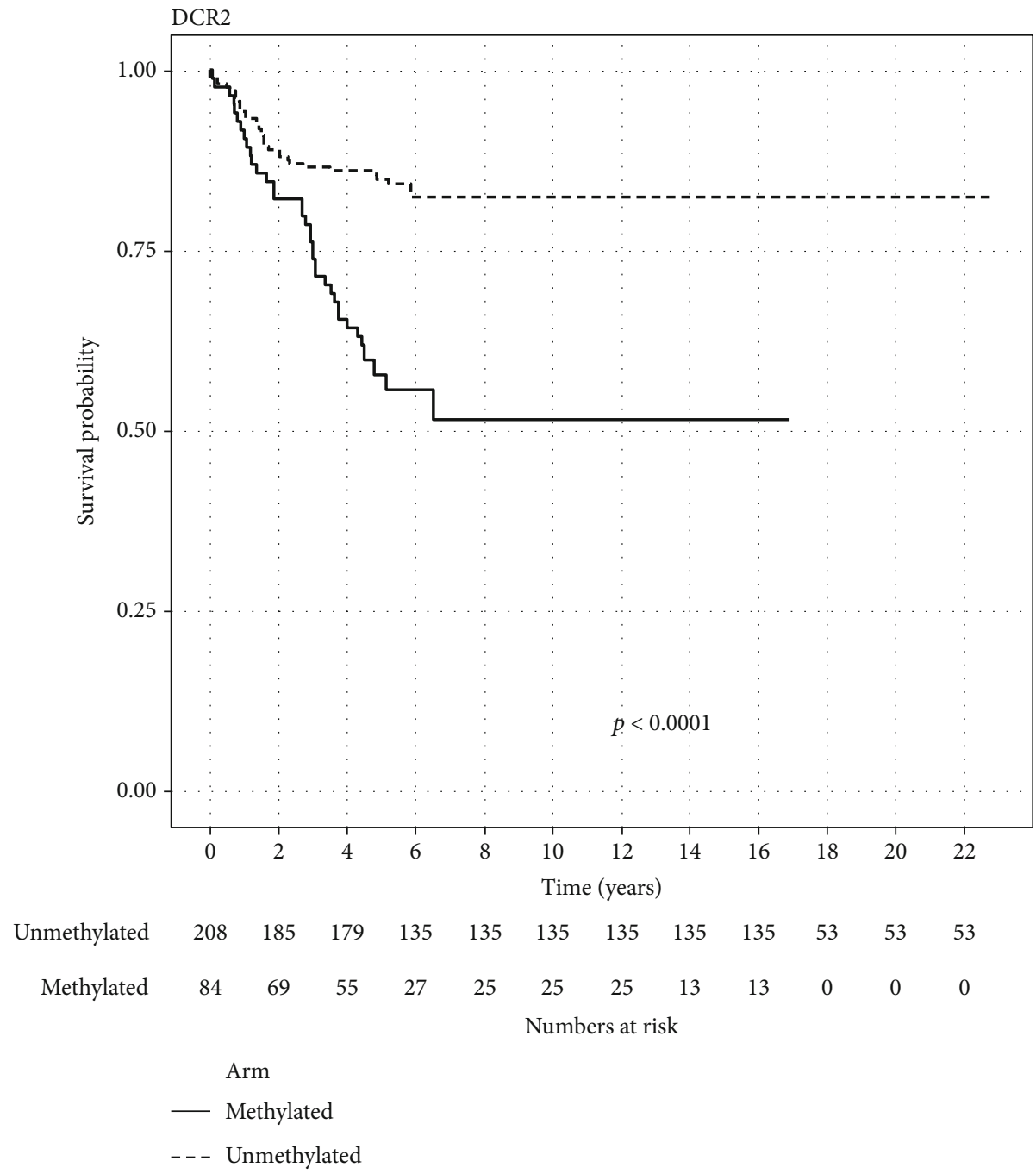

FIgURE 3: Reconstructed Kaplan-Meier graph of overall survival according to DCR2 methylation using aggregated data.

in our meta-analysis [21, 42], while two other studies (one of them is included in our meta-analysis) failed to demonstrate statistical significance perhaps due to small sample size or short follow-up duration [41,44]. The association between RASSF1A methylation and poor prognosis may be explained by its association with known poor prognostic factors such as older age, MYCN amplification, and high-risk group as previously discussed. This may not be entirely true as one study showed that patients with stage $4 \mathrm{NB}$ and older than 1 year having tumors with a high percentage of RASSF1A methylation $(>70 \%)$ had a significantly worse outcome than patients with similar prognostic criteria and a low percentage of RASSF1A methylation (5-year OS $19 \%$ vs. $56 \%$, respectively) [46]. Since RASSF1A may be correlated with other risk factors, multivariate analysis will be necessary to determine if RASSF1A is an independent prognostic factor. One study included in our meta-analysis conducted a multivariate analysis concluding that RASSF1A was an independent prognostic factor [42]. Interestingly, another study conducted multivariate analysis of survival including methylation of RASSF1A in serum as a prognostic variable. The hazard ratio of this analysis was 2.4 (95\% CI, 0.6-9.2); although this association did not reach statistical significance, these findings show that the methylation status of RASSF1A in the serum of patients with neuroblastoma may have the potential to become a prognostic predictor of outcome. This can be fostered by the results of the univariate analysis which showed that the influence of serum RASSF1A methylation on prognosis was comparable with that of the currently most reliable marker, MYCN amplification [43]. Most of other studies did not perform a multivariate analysis due to small sample size of included patients. Therefore, future studies will need to be performed with larger sample size to confirm our findings and to demonstrate if the RASSF1A can be used as an independent prognostic factor.

Some studies showed that RASSF1A expression can be restored in cell lines after treatment with the demethylating agent 5-Aza-dC [21, 47]. This may be considered a potential agent to be used in combination with chemotherapeutic drugs to treat hypermethylated RASSF1A neuroblastoma [48].

4.2. DCR2. DCR2 (decoy receptor 2) (TNRSF10D) is a tumor necrosis factor- $\alpha$ receptor family gene that is located 


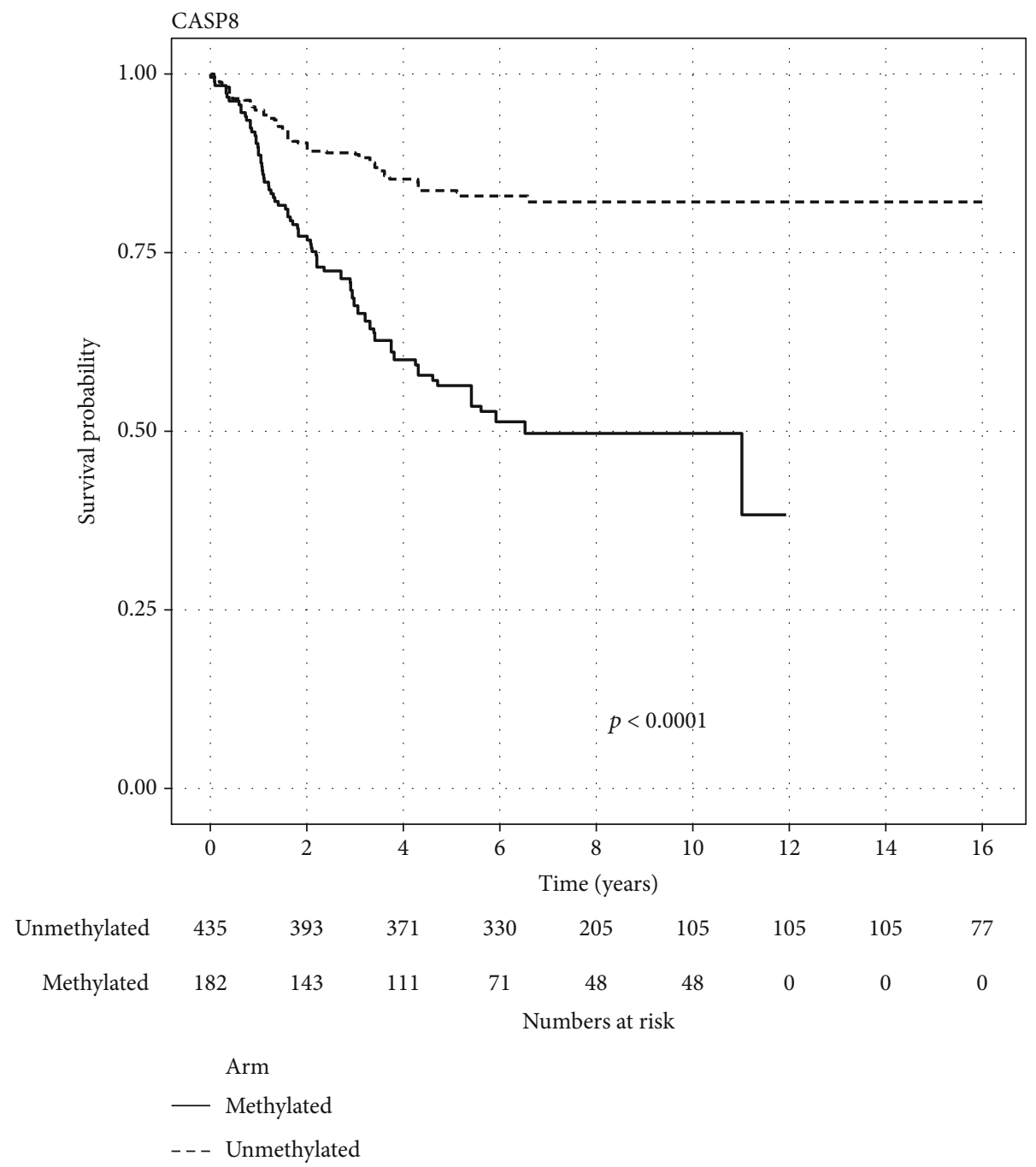

Figure 4: Reconstructed Kaplan-Meier graph of overall survival according to CASP8 methylation using aggregated data.

on 8 p21 $[44,49,50]$. The exact role that DCR2 plays in tumorigenesis remains unclear, as inactivation of DCR2 should have antitumor effects by sensitizing the cells to TRAIL-induced apoptosis [24]. However, the downregulation of DCR2 by promoter methylation is reported in various types of cancer $[42,49,50]$. One of the theories explaining this phenomenon is that it could be considered part of an inefficient defense mechanism activated to inhibit tumor cell growth $[23,44]$.

In our study, DCR2 was found methylated in $29 \%$ of NB patients; this was derived from the three studies included where methylation of DCR2 ranged from $21 \%$ to $44 \%$ [23, $42,49]$. Our result is similar to previous studies not included in this analysis, which have found DCR2 to be methylated in $25 \%-42 \%$ of NB tumor specimens $[41,44]$. One of the included studies in our analysis studied DCR2 methylation in serum and found that it was significantly correlated with DCR2 methylation in tumors $(r=0.67)$ [49]. Association between DCR2 methylation and other known prognostic factors has been tested in many previous studies. The frequency of DCR2 methylation was statistically significantly higher in high-risk groups compared to low-risk groups in one of the included studies in our meta-analysis [49]. This study further analyzed the frequency of DCR2 methylation focusing on patients with MYCN nonamplified tumors only. The prevalence of DCR2 methylation in the high-risk group was still statistically significantly higher than that in the low-risk group. Moreover, the frequency of DCR2 methylation between patients with MYCN amplified tumors was statistically significantly higher than that in patients with MYCN nonamplified tumors [49]. However, two other studies (one of them is included in our meta-analysis) concluded that DCR2 methylation is independent of MYCN amplification $[42,44]$. Another study stratified neuroblastoma patients according to whether the tumor sample was from a primary or a relapsed tumor. This study found that DCR2 was methylated in all $29 \%$ of relapsed tumors and in $25 \%$ of primary tumors, concluding that there is no change in the methylation profile at relapse [41]. Further studies with a larger number of patients may be needed to confirm these associations between DCR2 methylation and known risk factors.

In our study, 5-year survival probability for the DCR2 methylated arm was $57.78 \%$ (95\% CI $47.63-70.08$ ), while for the unmethylated arm, it was $84.84 \%$ (95\% CI 80.04 - 
89.92) (Figure 3) (log-rank $p$ value $<0.0001$ ). Adjusted HR for the methylated arm was 3.53 (95\% CI 2.20-5.65). These results are consistent with the results in all of the studies included in our meta-analysis and other previous studies $[23,42,44,49]$, while only one study failed to prove a statistically significant difference in OS between methylated and unmethylated DCR2 [41]. Two studies (one of them is included in our meta-analysis) analyzed the effect of DCR2 methylation in MYCN nonamplified tumors on overall survival. The first showed a trend for the negative prognostic effect of DCR2 methylation, failing to reach statistical significance mainly because of the small sample size in this cohort (17 patients) [44], while the included study was able to demonstrate statistical significance where patients with DCR2 methylation had a worse outcome than patients with unmethylated DCR2 (56\% versus 96\%, respectively; $p<$ $0.001)$ [49]. The same study tried to evaluate if an increase in serum DCR2 methylation can be used as an indicator to relapse. They measured serum DCR2 methylation in the clinical course of five patients. In two patients who achieved complete remission, serum DCR2 methylation decreased to an undetectable level, while in three patients who had recurrence after remission, the serum DCR2 methylation first decreased to an undetectable level and then increased again at the time of diagnosis [49]. In multivariate analysis to determine the prognostic effect of DCR2 methylation, two studies (one of them is included in our meta-analysis) failed to show that DCR2 methylation was a statistically significant independent prognostic factor probably due to the small sample size and large number of prognostic factors included in the model $[42,44]$, although one of these studies demonstrated that the combination of DCR2 and RASSF1A methylation was statistically significant in the multivariate model with RR 3.79 (95\% CI 1.01-14.22) [44]. The included study also found that DCR2 methylation was correlated with a poor outcome in children with a triploid, not diploid, tumor [42].

4.3. CASP8. CASP8 (located on human chromosome 2 band q33) methylation was first reported in neuroblastoma tumors nearly 18 years ago [51]. The CASP8 gene encodes a member of the cysteine-aspartic acid protease (caspase) family, which exerts its function inducing a death signaling complex through the Fas ligand pathway. The N-terminal FADDlike death effector domain of this protein suggests that it may interact with the Fas-interacting protein FADD [24, 36, 51-53]. Therefore, loss of expression or altered expression through methylation of this gene has been associated with the pathogenesis of variety of cancers such as neuroblastoma, medulloblastoma, lung cancer, and colorectal carcinomas [54-56].

In our study, CASP8 was found methylated in $30 \%$ of NB patients; this was derived from the five studies included where methylation of CASP8 ranged from $19 \%$ to $56 \%$ [23, $41,42,57,58]$. Our result is similar to previous studies not included in this analysis, which have found CASP8 to be methylated in $14 \%-62 \%$ of NB tumor specimens [36, 45, $51,59-62]$. There was only one study, which included 11 patients with stage $4 \mathrm{NB}$ and found that CASP8 is methylated in $91 \%$ of tumor samples. This study also measured CASP8 methylation in both tumors and bone marrow samples. They found that CASP8 methylation was found in 55\% of bone marrow samples [63].

Association between CASP8 methylation and known risk factors such as MYCN amplification, tumor stage, and age at diagnosis was studied in previous studies. There is a wide controversy in literature regarding the correlation between CASP8 methylation and MYCN amplification. Five studies (two of them are included in our meta-analysis) found that there was statistically significant correlation with CASP8 methylation and MYCN amplification as CASP8 methylation was more prevalent in MYCN amplified tumors $[42,45,51,57,62]$, while nine other studies (two of them are included in our meta-analysis) failed to prove this association concluding that CASP8 is not correlated with MYCN amplification [23, 36, 41, 44, 45, 61, 6466]. One meta-analysis was done to test the significance of this correlation, combining the results of three individual studies, which failed to demonstrate the statistical significance. The result was statistically significant with CASP8 methylation in $66 \%$ of MYCN amplified tumors versus $36 \%$ in MYCN nonamplified samples [45]. Similar controversy exists for the correlation between CASP8 and the stage of the disease. Three studies (two of them are included in our meta-analysis) were able to prove that CASP8 methylation was more prevalent in advanced stages $[41,57,62]$, while three other studies did not reach statistical significance for this correlation $[45,51,65]$. One study found that there was no statistically significant correlation between CASP8 methylation and age at diagnosis [65]. One study found that there was statistically significant correlation between RASSF1A methylation and CASP8 methylation [60]. Further studies with a larger number of patients may be needed to confirm these associations between CASP8 methylation and known risk factors.

In our study, 5-year survival probability for the CASP8 methylated arm was 56.39\% (95\% CI 49.53-64.19), while for the unmethylated arm, it was $83.68 \%$ (95\% CI 80.28 87.22) (Figure 4) (log-rank $p$ value $<0.0001)$. Adjusted HR for the methylated arm was 4.66 (95\% CI 3.42-6.35). These results are consistent with the results in the included studies in our analysis and other previous studies [23, 42, 45, 57, 58, 65]. Except for one study included in our meta-analysis which failed to prove a statistically significant difference between both arms, there was a numerical difference favoring the same trend [41]. In multivariate analysis to determine the prognostic effect of CASP8 methylation, two studies (one of them is included in our meta-analysis) showed that CASP8 methylation was an independent prognostic variable [57, 65]. The study included in our meta-analysis analyzed more genes; then, they grouped genes according to their cellular function to evaluate the effect of each group on the survival rates. Methylation of the apoptosis-related genes was the only group which showed a statistically significant effect on survival [57]. Another study included in our meta-analysis recruited patients from Germany and Japan. CASP8 methylation had a similar effect on survival in both populations [58]. In another study, the CASP8 protein expression effect on survival was evaluated in $140 \mathrm{NB}$ patients instead of 
CASP8 methylation. Surprisingly, CASP8 protein expression did not have an effect on survival [59].

Restoration of CASP8 expression in cell lines has been shown in previous studies to be necessary to restore TRAIL-mediated cell death [67-69]. IFN- $\gamma$ showed clinical evidence for restoration of CASP8 expression in NB patients in two previous small studies. However, there was no clinical correlation in these two studies $[70,71]$. Another study examined the combination of 5-dAzaC with IFN- $\gamma$ at relatively low individual drug doses. This combination was found to have a synergistic effect in CASP8 gene activation [72]. The molecular basis of this synergistic interaction of $5-\mathrm{dAzaC}$ and IFN- $\gamma$ may be explained by the different mechanisms of action of each agent to upregulate caspase-8. 5-dAzaC is a demethylating agent that reverses hypermethylation of a gene regulatory region of the caspase- 8 gene [73], whereas IFN- $\gamma$ enhances CASP8 levels through transcriptional activation of CASP8 through a Stat-1/IRF1-dependent pathway without altering the methylation status of the caspase- 8 gene [74]. Restoration of CASP8 expression in deficient neuroblastoma cells suppressed their metastases [75].

Our meta-analysis used IPD, as combining results of a survival time outcome using conventional methods such as pooling analysis from hazard ratios or time point outcomes is definitely challenging. One reason for that is that endpoints may be reported using different methods, as some studies may report HR while others may report median survival. Another reason would be that combining HRs from different studies may be subjected to bias, as these studies should check that the proportional hazard assumption is fulfilled. This assumption is seldom checked in most trials [76]. Several methods have been proposed earlier to extract data from published KM in order to carry out a meta-analysis. But most of these methods did not focus on the reconstruction of the KM or the life table data $[77,78]$. Therefore, the used algorithm developed by Guyot et al. was deemed the most appropriate for the purpose of this analysis [26]. This algorithm uses coordinates from KM extracted by digital software and the information on the initial number at risk and at different time points along with the number of events if available. The mean absolute error if all the information is available is certainly lower than that if only the initial number at risk is available. In studies included in our analysis, the number at risk at different time points and the number of events were not reported. The mean absolute error for determining survival probability if these data were not available is reported to be $0.328 \%$ (95\% CI $0.031 ; 2.233)$, while for HR, the mean absolute error is higher 0.198 (95\% CI $0.021 ; 1.556)$ [26]. Therefore, this is one of the limitations of our study, and thus, we encourage future studies with time-to-event outcomes to report numbers at risk at different points on the $\mathrm{KM}$ along with the number of events.

Other limitation of our study is that we pooled data from different populations over different covariates. We tried decreasing the impact of this by using multivariate Cox regression adjustment including the source of data as a covariate to adjust for this bias. Although the trend of results for the different papers included was generally similar, inter- pretation of our endpoints must be done with caution. In addition to that, our analysis used reconstructed IPD according to the methylation status only; as a result, we did not have any data for other risk factors. Hence, we were unable to perform multivariate analysis for the methylation of the studied genes on survival taking into account other known risk factors (e.g., MYCN amplification). Therefore, future large studies are needed to collect data about methylation of these genes along with other risk factors.

In conclusion, our results indicate that in NB patients, RASSF1A, DCR2, and CASP8 methylation is associated with poor prognosis. The fact that methylation of these genes may be reversible makes them a potential therapeutic target [79]. In addition, epigenetic alterations of these genes may be used as a marker of the disease. Large prospective studies will be necessary to confirm definitive correlation between methylation of these genes and survival outcome taking into account all other known risk factors.

\section{Disclosure}

This work is part of a Master's thesis of the Master's Program in Clinical Research, Center for Clinical Research and Management Education, Division of Health Care Sciences, Dresden International University, Dresden, Germany.

\section{Conflicts of Interest}

The authors declare that there is no conflict of interest regarding the publication of this paper.

\section{Acknowledgments}

Dr. Siepmann received grants from the Federal German Ministry of Health, the Kurt Goldstein Institute, and the Michael J. Fox Foundation that were not related to this study. Publication costs were funded by the Open Access Publication Funds of the Technische Universität Dresden.

\section{Supplementary Materials}

Supplementary Table 1: details of individual patient data retrieved. (Supplementary Materials)

\section{References}

[1] G. M. Brodeur, "Neuroblastoma: biological insights into a clinical enigma," Nature Reviews. Cancer, vol. 3, no. 3, pp. 203-216, 2003.

[2] S. Tsubota and K. Kadomatsu, "Origin and initiation mechanisms of neuroblastoma," Cell and Tissue Research, vol. 372, no. 2, pp. 211-221, 2018.

[3] E. Steliarova-Foucher, M. Colombet, L. A. G. Ries et al., "International incidence of childhood cancer, 2001-10: a populationbased registry study," The Lancet Oncology, vol. 18, no. 6, pp. 719-731, 2017.

[4] E. Ward, C. DeSantis, A. Robbins, B. Kohler, and A. Jemal, "Childhood and adolescent cancer statistics, 2014," CA: A Cancer Journal for Clinicians, vol. 64, no. 2, pp. 83-103, 2014. 
[5] F. Moreno, J. Lopez Marti, M. Palladino, P. Lobos, A. Gualtieri, and W. Cacciavillano, "Childhood neuroblastoma: incidence and survival in Argentina. Report from the National Pediatric Cancer Registry, ROHA Network 2000-2012," Pediatric Blood \& Cancer, vol. 63, no. 8, pp. 1362-1367, 2016.

[6] C. Spix, G. Pastore, R. Sankila, C. A. Stiller, and E. SteliarovaFoucher, "Neuroblastoma incidence and survival in European children (1978-1997): report from the Automated Childhood Cancer Information System project," European Journal of Cancer, vol. 42, no. 13, pp. 2081-2091, 2006.

[7] G. M. Brodeur, M. D. Hogarty, Y. P. Mosse, and J. M. Maris, Principles and practice of pediatric oncology, Lippincott, Williams and Wilkins, Philadelphia, 6th edition, 2011.

[8] N.-K. V. Cheung and M. A. Dyer, "Neuroblastoma: developmental biology, cancer genomics and immunotherapy," Nature Reviews Cancer, vol. 13, no. 6, pp. 397-411, 2013.

[9] R. A. Costa and H. N. Seuánez, "Investigation of major genetic alterations in neuroblastoma," Molecular Biology Reports, vol. 45, no. 3, pp. 287-295, 2018.

[10] A. M. Davidoff, "Neuroblastoma," Seminars in Pediatric Surgery, vol. 21, no. 1, pp. 2-14, 2012.

[11] N. R. Pinto, M. A. Applebaum, S. L. Volchenboum et al., "Advances in risk classification and treatment strategies for neuroblastoma," Journal of Clinical Oncology, vol. 33, no. 27, pp. 3008-3017, 2015.

[12] S. L. Cohn, A. D. J. Pearson, W. B. London et al., "The International Neuroblastoma Risk Group (INRG) classification system: an INRG Task Force report," Journal of Clinical Oncology, vol. 27, no. 2, pp. 289-297, 2009.

[13] J. M. Maris, M. D. Hogarty, R. Bagatell, and S. L. Cohn, "Neuroblastoma," The Lancet, vol. 369, no. 9579, pp. 2106-2120, 2007.

[14] J. M. Maris, "Recent advances in neuroblastoma," The New England Journal of Medicine, vol. 362, no. 23, pp. 2202-2211, 2010.

[15] A. Oberthuer, D. Juraeva, B. Hero et al., "Revised risk estimation and treatment stratification of low- and intermediate-risk neuroblastoma patients by integrating clinical and molecular prognostic markers," Clinical Cancer Research, vol. 21, no. 8, pp. 1904-1915, 2015.

[16] B. Hero, T. Simon, R. Spitz et al., "Localized infant neuroblastomas often show spontaneous regression: results of the prospective trials NB95-S and NB97," Journal of Clinical Oncology, vol. 26, no. 9, pp. 1504-1510, 2008.

[17] D. L. Baker, M. L. Schmidt, S. L. Cohn et al., "Outcome after reduced chemotherapy for intermediate-risk neuroblastoma," New England Journal of Medicine, vol. 363, no. 14, pp. 13131323, 2010.

[18] S. Modak, B. H. Kushner, M. P. LaQuaglia, K. Kramer, and N.K. V. Cheung, "Management and outcome of stage 3 neuroblastoma," European Journal of Cancer, vol. 45, no. 1, pp. 90-98, 2009.

[19] I. Øra and A. Eggert, "Progress in treatment and risk stratification of neuroblastoma: impact on future clinical and basic research," Seminars in Cancer Biology, vol. 21, no. 4, pp. 217-228, 2011.

[20] M. Olsson, S. Beck, P. Kogner, T. Martinsson, and H. Carén, "Genome-wide methylation profiling identifies novel methylated genes in neuroblastoma tumors," Epigenetics, vol. 11, no. 1, pp. 74-84, 2016.
[21] Q. Yang, P. Zage, D. Kagan et al., “Association of epigenetic inactivation of RASSF1A with poor outcome in human neuroblastoma," Clinical Cancer Research, vol. 10, no. 24, pp. 84938500, 2004.

[22] R. Domingo-Fernandez, K. Watters, O. Piskareva, R. L. Stallings, and I. Bray, "The role of genetic and epigenetic alterations in neuroblastoma disease pathogenesis," Pediatric Surgery International, vol. 29, no. 2, pp. 101-119, 2013.

[23] Q. Yang, C. M. Kiernan, Y. Tian et al., "Methylation of CASP8, DCR2, and HIN-1 in neuroblastoma is associated with poor outcome," Clinical Cancer Research, vol. 13, no. 11, pp. 3191-3197, 2007.

[24] A. Decock, M. Ongenaert, J. Vandesompele, and F. Speleman, "Neuroblastoma epigenetics: from candidate gene approaches to genome-wide screenings," Epigenetics, vol. 6, no. 8, pp. 962-970, 2014.

[25] "ScanIt," Amsterchem V 2.07, https://www.amsterchem.com/ scanit.html.

[26] P. Guyot, A. E. Ades, M. J. N. M. Ouwens, and N. J. Welton, "Enhanced secondary analysis of survival data: reconstructing the data from published Kaplan-Meier survival curves," BMC Medical Research Methodology, vol. 12, no. 1, p. 9, 2012.

[27] R. Core Team, $R$ : a language and environment for statistical computing, R Foundation for Statistical Computing, Vienna, Austria, 2014, http://www.R-project.org/.

[28] S. Sharma, T. K. Kelly, and P. A. Jones, "Epigenetics in cancer," Carcinogenesis, vol. 31, no. 1, pp. 27-36, 2010.

[29] P. A. Jones and S. B. Baylin, "The fundamental role of epigenetic events in cancer," Nature Reviews. Genetics, vol. 3, no. 6, pp. 415-428, 2002.

[30] K. Grønbaek, C. Hother, and P. A. Jones, "Epigenetic changes in cancer," APMIS, vol. 115, no. 10, pp. 1039-1059, 2007.

[31] S. B. Baylin, "DNA methylation and gene silencing in cancer," Nature Clinical Practice Oncology, vol. 2, no. S1, pp. S4-S11, 2005.

[32] F. F. Caputi, S. Candeletti, and P. Romualdi, "Epigenetic Approaches in Neuroblastoma Disease Pathogenesis," in Neuroblastoma - Current State and Recent Updates, InTech, 2017.

[33] G. P. Tonini and M. Romani, "Genetic and epigenetic alterations in neuroblastoma," Cancer Letters, vol. 197, no. 1-2, pp. 69-73, 2003.

[34] M. Alaminos, V. Davalos, N. K. V. Cheung, W. L. Gerald, and M. Esteller, "Clustering of gene hypermethylation associated with clinical risk groups in neuroblastoma," JNCI: Journal of the National Cancer Institute, vol. 96, no. 16, pp. 1208-1219, 2004.

[35] R. Dammann, C. Li, J. H. Yoon, P. L. Chin, S. Bates, and G. P. Pfeifer, "Epigenetic inactivation of a RAS association domain family protein from the lung tumour suppressor locus 3p21.3," Nature Genetics, vol. 25, no. 3, pp. 315-319, 2000.

[36] P. Lázcoz, J. Muñoz, M. Nistal, Á. Pestaña, I. Encío, and J. S. Castresana, "Frequent promoter hypermethylation of RASSF1A and CASP8 in neuroblastoma," BMC Cancer, vol. 6, no. 1, p. 254, 2006.

[37] L. Shivakumar, J. Minna, T. Sakamaki, R. Pestell, and M. A. White, "The RASSF1A tumor suppressor blocks cell cycle progression and inhibits cyclin D1 accumulation," Molecular and Cellular Biology, vol. 22, no. 12, pp. 4309-4318, 2002.

[38] K. Harada, S. Toyooka, A. Maitra et al., "Aberrant promoter methylation and silencing of the RASSF1A gene in pediatric 
tumors and cell lines," Oncogene, vol. 21, no. 27, pp. 43454349, 2002.

[39] R. Dammann, G. Yang, and G. P. Pfeifer, "Hypermethylation of the cpG island of Ras association domain family $1 \mathrm{~A}$ (RASSF1A), a putative tumor suppressor gene from the 3p21.3 locus, occurs in a large percentage of human breast cancers," Cancer Research, vol. 61, no. 7, pp. 3105-3109, 2001.

[40] J. H. Yoon, R. Dammann, and G. P. Pfeifer, "Hypermethylation of the CpG island of the RASSF1A gene in ovarian and renal cell carcinomas," International Journal of Cancer, vol. 94, no. 2, pp. 212-217, 2001.

[41] M. B. Michalowski, F. de Fraipont, D. Plantaz, S. Michelland, V. Combaret, and M. C. Favrot, "Methylation of tumorsuppressor genes in neuroblastoma: the RASSF1A gene is almost always methylated in primary tumors," Pediatric Blood \& Cancer, vol. 50, no. 1, pp. 29-32, 2008.

[42] M. Haruta, T. Kamijo, A. Nakagawara, and Y. Kaneko, "RASSF1A methylation may have two biological roles in neuroblastoma tumorigenesis depending on the ploidy status and age of patients," Cancer Letters, vol. 348, no. 1-2, pp. 167-176, 2014.

[43] A. Misawa, S. Tanaka, S. Yagyu et al., "RASSF1A hypermethylation in pretreatment serum DNA of neuroblastoma patients: a prognostic marker," British Journal of Cancer, vol. 100, no. 2, pp. 399-404, 2009.

[44] B. Banelli, I. Gelvi, A. di Vinci et al., "Distinct CpG methylation profiles characterize different clinical groups of neuroblastic tumors," Oncogene, vol. 24, no. 36, pp. 5619-5628, 2005.

[45] J. Hoebeeck, E. Michels, F. Pattyn et al., "Aberrant methylation of candidate tumor suppressor genes in neuroblastoma," Cancer Letters, vol. 273, no. 2, pp. 336-346, 2009.

[46] J. Stutterheim, F. A. Ichou, E. den Ouden et al., "Methylated RASSF1a is the first specific DNA marker for minimal residual disease testing in neuroblastoma," Clinical Cancer Research, vol. 18, no. 3, pp. 808-814, 2012.

[47] G. P. Pfeifer and R. Dammann, "Methylation of the tumor suppressor gene RASSF1A in human tumors," Biochemistry, vol. 70, no. 5, pp. 576-583, 2005.

[48] J. Charlet, M. Schnekenburger, K. W. Brown, and M. Diederich, "DNA demethylation increases sensitivity of neuroblastoma cells to chemotherapeutic drugs," Biochemical Pharmacology, vol. 83, no. 7, pp. 858-865, 2012.

[49] S. Yagyu, T. Gotoh, T. Iehara et al., "Circulating methylated-DCR2 gene in serum as an indicator of prognosis and therapeutic efficacy in patients with MYCN nonamplified neuroblastoma," Clinical Cancer Research, vol. 14, no. 21, pp. 7011-7019, 2008

[50] M. M. van Noesel, S. van Bezouw, G. S. Salomons et al., "Tumor-specific down-regulation of the tumor necrosis factor-related apoptosis-inducing ligand decoy receptors DcR1 and DcR2 is associated with dense promoter hypermethylation," Cancer Research, vol. 62, no. 7, pp. 2157-2161, 2002.

[51] T. Teitz, T. Wei, M. B. Valentine et al., "Caspase 8 is deleted or silenced preferentially in childhood neuroblastomas with amplification of MYCN," Nature Medicine, vol. 6, no. 5, pp. 529-535, 2000.

[52] B. B. Wolf and D. R. Green, "Suicidal tendencies: apoptotic cell death by caspase family proteinases," The Journal of Biological Chemistry, vol. 274, no. 29, pp. 20049-20052, 1999.
[53] H. Steller, "Artificial death switches: induction of apoptosis by chemically induced caspase multimerization," Proceedings of the National Academy of Sciences of the United States of America, vol. 95, no. 10, pp. 5421-5422, 1998.

[54] N. Shivapurkar, S. Toyooka, M. T. Eby et al., "Differential inactivation of caspase- 8 in lung cancers," Cancer Biology \& Therapy, vol. 1, no. 1, pp. 65-69, 2002.

[55] C. Pingoud-Meier, D. Lang, A. J. Janss et al., "Loss of caspase-8 protein expression correlates with unfavorable survival outcome in childhood medulloblastoma," Clinical Cancer Research, vol. 9, no. 17, pp. 6401-6409, 2003.

[56] H. S. Kim, J. W. Lee, Y. H. Soung et al., "Inactivating mutations of caspase- 8 gene in colorectal carcinomas," Gastroenterology, vol. 125, no. 3, pp. 708-715, 2003.

[57] E. Grau, F. Martinez, C. Orellana et al., "Hypermethylation of apoptotic genes as independent prognostic factor in neuroblastoma disease," Molecular Carcinogenesis, vol. 50, no. 3, pp. 153-162, 2011.

[58] K. Asada, N. Watanabe, Y. Nakamura et al., "Stronger prognostic power of the $\mathrm{CpG}$ island methylator phenotype than methylation of individual genes in neuroblastomas," Japanese Journal of Clinical Oncology, vol. 43, no. 6, pp. 641-645, 2013.

[59] S. Fulda, C. Poremba, B. Berwanger et al., "Loss of caspase-8 expression does not correlate withMYCNAmplification, aggressive disease, or prognosis in neuroblastoma," Cancer Research, vol. 66, no. 20, pp. 10016-10023, 2006.

[60] D. Astuti, A. Agathanggelou, S. Honorio et al., "RASSF1A promoter region $\mathrm{CpG}$ island hypermethylation in phaeochromocytomas and neuroblastoma tumours," Oncogene, vol. 20, no. 51, pp. 7573-7577, 2001.

[61] N. B. Kiss, P. Kogner, J. I. Johnsen, T. Martinsson, C. Larsson, and J. Geli, "Quantitative global and gene-specific promoter methylation in relation to biological properties of neuroblastomas," BMC Medical Genetics, vol. 13, no. 1, p. 83, 2012.

[62] P. Gonzalez-Gomez, M. J. Bello, J. Lomas et al., "Aberrant methylation of multiple genes in neuroblastic tumours," European Journal of Cancer, vol. 39, no. 10, pp. 1478-1485, 2003.

[63] E. Grau, F. Martinez, C. Orellana et al., "Epigenetic alterations in disseminated neuroblastoma tumour cells: influence of TMS1 gene hypermethylation in relapse risk in NB patients," Journal of Cancer Research and Clinical Oncology, vol. 136, no. 9, pp. 1415-1421, 2010.

[64] J. Takita, H. W. Yang, Y. Y. Chen et al., "Allelic imbalance on chromosome $2 \mathrm{q}$ and alterations of the caspase 8 gene in neuroblastoma," Oncogene, vol. 20, 32 pages, 2001.

[65] A. Rihani, B. de Wilde, F. Zeka et al., "CASP8 SNP D302H (rs1045485) is associated with worse survival in MYCNamplified neuroblastoma patients," PLoS One, vol. 9, no. 12, article e114696, 2014.

[66] K. Harada, S. Toyooka, N. Shivapurkar et al., "Deregulation of caspase 8 and 10 expression in pediatric tumors and cell lines," Cancer Research, vol. 62, no. 20, pp. 5897-5901, 2002.

[67] A. Mühlethaler-Mottet, K. B. Bourloud, K. Auderset, J. M. Joseph, and N. Gross, "Drug-mediated sensitization to TRAIL-induced apoptosis in caspase-8-complemented neuroblastoma cells proceeds via activation of intrinsic and extrinsic pathways and caspase-dependent cleavage of XIAP, Bcl-xL and RIP," Oncogene, vol. 23, no. 32, pp. 5415-5425, 2004.

[68] A. Mühlethaler-Mottet, K. Balmas, K. Auderset, J.-M. Joseph, and N. Gross, "Restoration of TRAIL-induced apoptosis in a caspase-8-deficient neuroblastoma cell line by stable re- 
expression of caspase-8," Annals of the New York Academy of Sciences, vol. 1010, no. 1, pp. 195-199, 2003.

[69] S. Kim, J. Kang, B. M. Evers, and D. H. Chung, "Interferon- $\gamma$ induces caspase- 8 in neuroblastomas without affecting methylation of caspase- 8 promoter," Journal of Pediatric Surgery, vol. 39, no. 4, pp. 509-515, 2004.

[70] A. E. McKee and C. J. Thiele, "Targeting caspase 8 to reduce the formation of metastases in neuroblastoma," Expert Opinion on Therapeutic Targets, vol. 10, no. 5, pp. 703-708, 2006.

[71] H.-x. Tong, C.-w. Lu, J.-h. Zhang, and J.-h. Zhang, "Enhanced effect of IFN $\gamma$ on the induced apoptosis of neuroblastoma cells by cytotoxic drugs," Pediatric Hematology and Oncology, vol. 25, no. 6, pp. 549-558, 2009.

[72] S. Fulda and K.-M. Debatin, “5-Aza-2'-deoxycytidine and IFN- $\gamma$ cooperate to sensitize for TRAIL-induced apoptosis by upregulating caspase-8," Oncogene, vol. 25, no. 37, pp. 51255133, 2006.

[73] S. Fulda, M. U. Küfer, E. Meyer, F. van Valen, B. DockhornDworniczak, and K.-M. Debatin, "Sensitization for death receptor- or drug-induced apoptosis by re-expression of caspase- 8 through demethylation or gene transfer," Oncogene, vol. 20, no. 41, pp. 5865-5877, 2001.

[74] S. Fulda, E. Meyer, and K. M. Debatin, "Inhibition of TRAILinduced apoptosis by Bcl-2 overexpression," Oncogene, vol. 21, no. 15, pp. 2283-2294, 2002.

[75] D. G. Stupack, T. Teitz, M. D. Potter et al., "Potentiation of neuroblastoma metastasis by loss of caspase-8," Nature, vol. 439, no. 7072, pp. 95-99, 2006.

[76] P. Guyot, N. J. Welton, M. J. N. M. Ouwens, and A. E. Ades, "Survival time outcomes in randomized, controlled trials and meta-analyses: the parallel universes of efficacy and cost-effectiveness," Value Health, vol. 14, no. 5, pp. 640-646, 2011.

[77] T. P. A. Debray, K. G. M. Moons, G. van Valkenhoef et al., "Get real in individual participant data (IPD) meta-analysis: a review of the methodology," Research Synthesis Methods, vol. 6, no. 4, pp. 293-309, 2015.

[78] S. Katsahian, A. Latouche, J.-Y. Mary, S. Chevret, and R. Porcher, "Practical methodology of meta-analysis of individual patient data using a survival outcome," Contemporary Clinical Trials, vol. 29, no. 2, pp. 220-230, 2008.

[79] L. Jubierre, C. Jiménez, E. Rovira et al., "Targeting of epigenetic regulators in neuroblastoma," Experimental \& Molecular Medicine, vol. 50, no. 4, p. 51, 2018. 